\title{
The development of a nurse-driven protocol for the safe administration of vasopressors through peripheral intravenous catheters
}

\author{
Laura Padrone ${ }^{1}$, Janet Galiczewski*1,2, Bernadette Amitrano ${ }^{1}$, Seth Koenig ${ }^{1,3}$, Mangala Narasimhan ${ }^{1,3}$, Paul H. \\ Mayo $^{3,4}$ \\ ${ }^{1}$ The Medical Intensive Care Unit at Northwell Health: Long Island Jewish Medical Center, New York, United States \\ ${ }^{2}$ School of Nursing, Stony Brook University, New York, United States \\ ${ }^{3}$ Hofstra Northwell School of Medicine, New York, United States \\ ${ }^{4}$ Pulmonary, Critical Care, and Sleep Medicine at Northwell Health: Long Island Jewish Medical Center, New York, United States
}

Received: December 5, 2017

DOI: $10.5430 /$ ijh.v4n1p36
Accepted: February 22, $2018 \quad$ Online Published: March 8, 2018

URL: https://doi.org/10.5430/ijh.v4n1p36

\begin{abstract}
Aim: The purpose of this quality improvement project was to develop a nurse-driven protocol to provide a safer, quicker, and less complication-prone alternative for the administration of vasopressors.

Background: The use of vasopressor medication is a common life saving intervention used to achieve hemodynamic stability in critically ill patients. Vasopressor infusions are routinely administered through central venous catheters (CVC) for fear that extravasation into subcutaneous tissue will result in ischemic injury. There are many potential complications of obtaining central venous access for vasopressor use, including pneumothoraces, arterial punctures and hematomas.

Design: Quality improvement project.

Methods: An interdisciplinary team developed a written protocol for the administration of vasopressors through peripheral intravenous (PIV) catheters based on the available scientific evidence. Key components of this quality improvement project were patient safety, a team approach and skilled monitoring of the infusion site. Critical care nurses were responsible for the line insertion, maintenance and identification of possible extravasation. All catheters placed were confirmed by ultrasonography and if extravasation of medication was suspected, the extravasation protocol was followed without delay.

Results: The protocol developed was utilized in a study by Cardenas-Garcia et al. (2015). Extravasation occurred in 19 of 734 patients $(2 \%)$ without any tissue injury following the use of the extravasation protocol. This evidence-based protocol for peripherally administered vasopressors decreased central line use and the potential complications from the placement of CVCs. Strict adherence to this evidence-based nurse-driven protocol was essential for the safe administration of vasopressors via PIV access.

Conclusion/Clinical relevance: Peripheral administration of vasopressors has many beneficial implications for nursing practice. A nurse-driven protocol for peripheral vasopressors can eliminate the need for CVCs, which will reduce the incidence of central line-associated blood stream infections and decrease the number of central line days. This also may eliminate potential complications from CVC placement. Lastly, peripherally administered vasopressors may prevent a delay in treatment often experienced with placement of a central line.
\end{abstract}

Key Words: Nurse-driven protocol, Peripherally administered vasopressors, Intensive care unit

*Correspondence: Janet Galiczewski; Email: janet.galiczewski@ stonybrook.edu; Address: Stony Brook University, School of Nursing, HSC Level 2-204, Stony Brook, New York, United States. 


\section{INTRODUCTION}

The use of vasopressors is a common life saving intervention used to achieve hemodynamic stability in critically ill patients. Vasopressor infusions have traditionally been administered through central venous catheter (CVC) access via the subclavian, internal jugular or femoral veins. Peripheral intravenous (PIV) catheters have long been avoided for fear that extravasation into the subcutaneous tissue will result in local ischemic injury. However, central venous access is associated with numerous complications, such as central line-associated blood stream infections (CLABSI), procedural complications, and it may not be practical in an emergency situation. ${ }^{[1]}$ Additionally, peripherally inserted central line catheters (PICC) are associated with catheter fracture, upper extremity venous thrombosis, pulmonary embolism and sepsis. ${ }^{[2]}$

\subsection{Background and significance}

Procedural complications such as pneumothoraces, arterial punctures, and hematomas occur in approximately $15 \%$ of patients during the insertion of CVCs. ${ }^{[3]}$ Furthermore, over 60,000 CLABSIs occur in United States hospitals annually at a cost of $\$ 1.85$ billion dollars. ${ }^{[4]}$ Globally, this cost is even higher and it negatively affects patient outcomes. A review of the literature and inconsistencies with practice guidelines led to the creation of a task force in an 18-bed medical intensive care unit (MICU) of a tertiary academic medical center in New York. The task force consisted of critical care nurses, physicians and pharmacists. Their charge was to re-evaluate the safety of administration of vasopressors via PIV access. The purpose of this quality improvement project was to develop a nurse-driven protocol to provide a safer, quicker, and less complication-prone alternative for the administration of vasopressors.

\subsection{Review of the literature}

Protocols are used to implement evidence-based interventions that improve patient outcomes and the overall quality of care. ${ }^{[5]}$ Standardization of health care and the use of protocol-based care minimizes complications and positively affects delivery of care. ${ }^{[6,7]}$ Protocols are developed by interdisciplinary healthcare teams and are based on review of the scientific evidence. ${ }^{[8]}$ Measuring protocol-based care is often difficult. Patient outcomes, reduced length of stay and reduction of complications are used to determine the effectiveness of the standardization of care. ${ }^{[9]}$

A review of the literature yielded few studies that implemented a nurse-driven protocol for administration of vasopressors through PIV access. A retrospective chart review of vasopressor administration through a PIV catheter demonstrated a $4 \%$ overall incidence of extravasation events when

Published by Sciedu Press a strict safety protocol was not in place. ${ }^{[10]}$ None of these events were severe enough to require intervention and vasopressors were resumed in a new peripheral site most of the time. ${ }^{[10]}$ This study suggested that the administration of vasopressors through a CVC may not be a necessity for all patients. $^{[10]}$

In a 6-month pilot study, phenylephrine was administered via PIV access in 20 patients with one minor complication and no major injuries. ${ }^{[11]}$ This was achieved by implementing safety measures in the computerized physician order entry system (CPOE) and by utilizing nurse-driven protocols. This study concluded that with careful monitoring and safety protocols, peripheral administration of vasopressors may be acceptable. ${ }^{[11]}$

A systematic review of the literature conducted by Loubani and Green ${ }^{[12]}$ determined that vasopressors can cause tissue damage and limb injury when given via PIV access. Case reports demonstrated that administration of vasopressors greater than 2 hours and placement of the PIV catheter distal to the antecubital fossa were more likely to cause local tissue injury. ${ }^{[12]}$ Overall, no conclusion can be drawn from this systemic review regarding the safety of vasopressors via PIV access. ${ }^{[12]}$

Kahn et al. ${ }^{[13]}$ reported a case study of a 46-year-old female with septic shock and severe hypotension. Vasopressin was started via an 18-gauge PIV catheter in her wrist. She subsequently developed a necrotic tissue injury proximal to the PIV site. This protocol called for an assessment of the PIV site with vasopressor administration every 4 hours. ${ }^{[13]}$ The literature states that progressive tissue damage after extravasation of vasopressin continues even after the infusion is discontinued, and therefore, PIV administration is not recommended. ${ }^{[13]}$

Le \& Patel ${ }^{[14]}$ conducted a systematic review of the literature on the extravasation of noncytotoxic drugs. The review consisted of some vasoactive agents, such as dopamine, epinephrine, norepinephrine and vasopressin. These drugs can cause skin inflammation, pain and possible tissue necrosis. Extravasation of vasoactive drugs, if left untreated, can cause significant morbidity and loss of limb. ${ }^{[14]}$ This can increase length of stay and healthcare costs, as well as making the institution vulnerable to litigation. ${ }^{[15]}$ When extravasation occurs, the infusion must immediately be discontinued. The remaining medication in the catheter must be aspirated to prevent further leakage into the tissue. Prompt treatment with an antidote should be initiated. ${ }^{[14]}$ Having a standard of care for prevention of extravasation that includes astute and frequent assessment of the site is imperative before clinicians decide to administer vasopressors via a PIV. ${ }^{[14-16]}$ 


\section{THE DEVELOPMENT OF A NURSE-DRIVEN PROTOCOL}

After review of the literature, the task force developed a written protocol for administration of vasopressors through peripheral catheters (see Table 1). The task force was comprised of three full-time critical care intensivists, critical care and pulmonary fellows, a clinical pharmacist, nursing leadership, nursing education and critical care certified clinical nurses. The protocol was approved by the department of pharmacy and the hospital therapeutics committee. The overall process took approximately 6 months. Prior to imple- mentation, the nursing and medical staff received in-service training on the protocol and recognition of extravasation. Infusion Nursing Standards of Practice defines extravasation as "the inadvertent administration of a vesicant medication or solution into the surrounding tissue rather than into the intended vascular pathway". ${ }^{[15,17]}$ This broad definition does not distinguish extravasation from infiltration. For the purpose of this quality improvement study, the authors define extravasation as occurring when the PIV was placed correctly into the lumen of the vessel (confirmed by ultrasonography) but due to injury of the vessel wall, the fluid leaked out into the surrounding tissues. ${ }^{[15]}$

Table 1. Adult guidelines for the peripheral administration of vasopressor therapy in the intensive care unit (ICU)

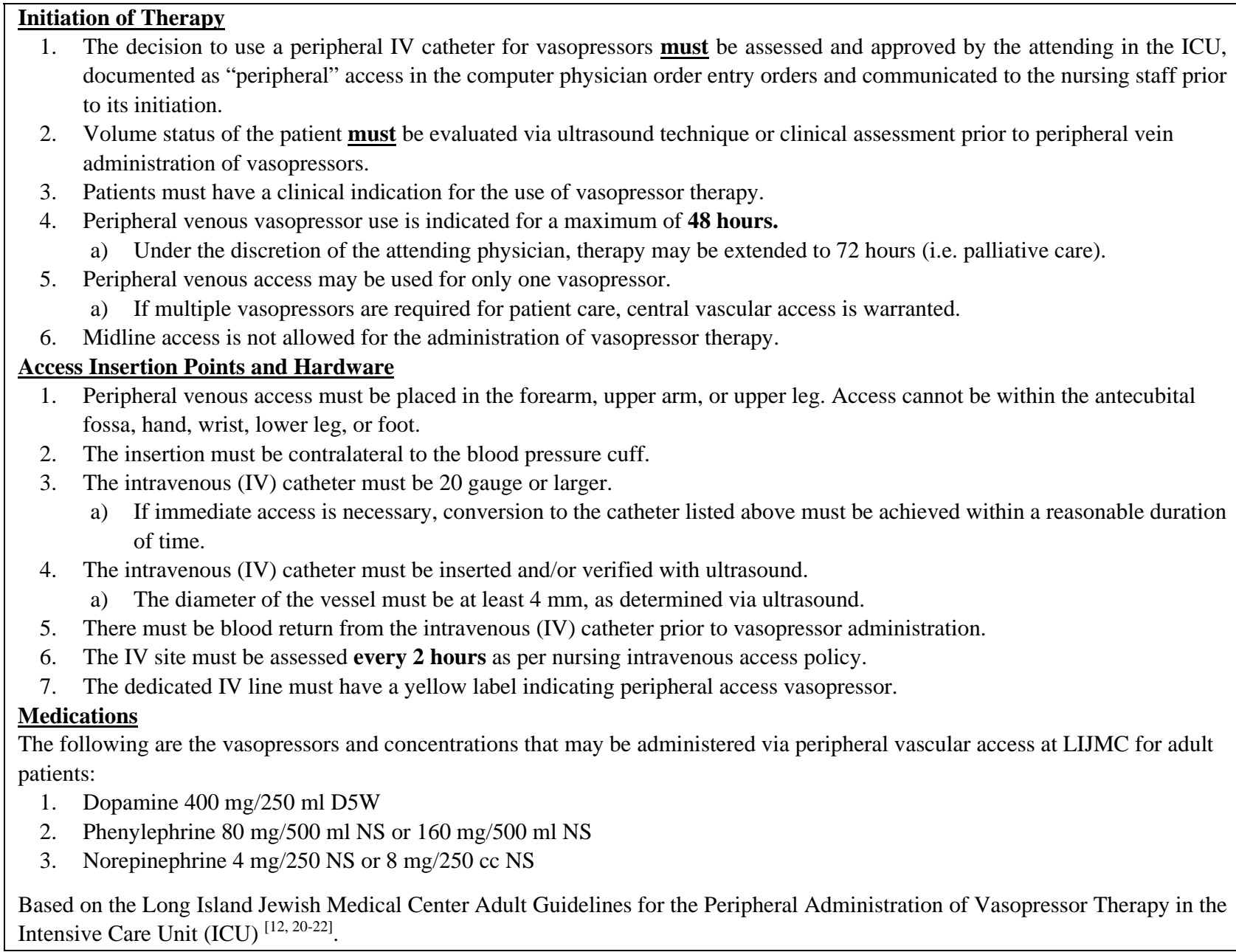

The decision to administer peripheral vasopressors was made by the clinical management team of the MICU. Peripheral vasopressor use required a PIV access to be placed in the upper arm or forearm. Access could not be within the antecubital fossa, hand, wrist, lower leg, or foot for fear that these regions may be prone to extravasation due to their anatomical locations. ${ }^{[14,15]}$ Extravasation of vasopressor medications can cause cell damage by inducing arterial and peripheral vasoconstriction at the site. ${ }^{[14]}$ Requirements also included a vessel diameter of $4 \mathrm{~mm}$ or greater, as measured by ultrasonography and the use of a large bore PIV catheter gauge 20 or greater. ${ }^{[18]}$ PIV access could only be used for one vasopressor at a time. If multiple vasopressors were required for patient care, central vascular access was warranted. A PIV 
catheter could be placed without the use of ultrasonography, but did require confirmation via ultrasonography prior to initiation of vasopressor agents. ${ }^{[19]}$ Midline catheters were not included in this protocol because there may be delays in detecting the signs and symptoms of extravasation due to where these lines terminate.

Peripheral vasopressor administration was allowed for 48 hours and was extended up to 72 hours at the discretion of the attending physician. ${ }^{[12]}$ Non-invasive blood pressure monitoring was required to be contralateral to the arm with the PIV access. The following maximum concentrations of vasopressors were deemed acceptable to infuse via PIV access: norepinephrine $8 \mathrm{mg}$ in $250 \mathrm{ml}$ of normal saline, phenylephrine $160 \mathrm{mg}$ in $500 \mathrm{ml}$ of normal saline, and dopamine $400 \mathrm{mg}$ in $250 \mathrm{ml}$ of D5W. ${ }^{[14]}$ Vasopressin was not administered peripherally as there is no reversal agent. ${ }^{[14]} \mathrm{A}$ provider's order indicating that a PIV catheter has been placed or verified by ultrasonography and was acceptable for vasopressor use was required, along with an explicit statement of its location and gauge. The dedicated PIV access was tagged with a yellow label to clearly indicate peripheral vasopressor use.
The essential safety element of this protocol was frequent assessment of the integrity of the PIV catheter and access site by nursing staff. The PIV catheter was assessed for blood return and patency at a minimum of every 2 hours on all shifts and documented in the electronic medical record (EMR) in the patient access section. During change of shift, clinical nurses assessed the PIV site and documentation together. A nursing brief was conducted at the beginning of every shift and protocol adherence was addressed. The nurse managers conducted chart audits monthly. PIV site skin integrity and blood return were consistently documented as per protocol. Immediate provider notification was required if there was concern for extravasation and the extravasation protocol was initiated (see Table 2). In the event that blood return was no longer present, confirmation of placement of the current PIV catheter via ultrasound by a physician was required in order to ensure that extravasation had not occurred. Prompt recognition of extravasation and initiation of the reversal agents were vital in the prevention of long-term complications. ${ }^{[14,23]}$ The nursing and medical teams were educated on the protocol prior to its initiation, with emphasis on strict adherence to ensure patient safety.

Table 2. Extravasation protocol

Adult Guidelines for the Extravasation of Peripheral Vasopressors in the Critical Care Units

The following is the procedure to follow if extravasation of vasopressors has occurred.

\section{Extravasation Treatment with Phentolamine}

1. On suspecting extravasation, the infusion must be stopped immediately.

2. The prescriber must be contacted immediately in order to assess the site and initiate treatment.

3. The IV catheter is left in place and any residual medication is aspirated through the catheter. The IV catheter is then removed.

4. The extent of the extravasation is marked with a pen to provide a baseline for monitoring.

5. 1 vial of $\mathbf{5} \mathbf{~ m g}$ of Phentolamine administered as follows:

a) $10 \mathrm{ml}$ of normal saline is added to the vial to reconstitute phentolamine powder to a final concentration of $0.5 \mathrm{mg} / \mathrm{ml}$.

b) After full dissolution, the contents of the vial are loaded into one $10 \mathrm{ml}$ syringe.

c) 25 or $\mathbf{2 7}$ gauge needles are used for each injection, and the needle should be changed after each injection. Therefore, 5 needles are needed.

d) Inject the $10 \mathrm{ml}$ of phentolamine subcutaneously into the affected area as 5 separate $2 \mathrm{ml}$ clockwise injections around the leading edge of the extravasation marked by the pen.

e) At the judgment of the attending or fellow in the ICU, $2.5 \mathbf{~ c m}$ of nitroglycerin paste may be applied to the area of extravasation.

6. A medication occurrence must be filled out and sent to the proper departments for review.

Based on the Long Island Jewish Medical Center Adult Guidelines for the Extravasation of Peripheral Vasopressors in the Critical Care Units ${ }^{[12,20,22]}$.

\section{RESULTS OF IMPLEMENTATION NURSE-DRIVEN PROTOCOL}

A well-documented study by Cardenas-Garcia et al. (2015) utilized this nurse-driven protocol in an 18-bed MICU in a tertiary care teaching medical center in New York. Data was

Published by Sciedu Press

(
OF collected over a 20-month time period on a large cohort of hypotensive patients. Data collection included patient demographics, duration of vasopressor use, dosage of vasopressors, route of administration, extravasation of vasopressors, conversion to CVC, and patient outcomes, such as mortality, 
length of stay, and limb injury. Seven hundred and thirty four patients (male/female 398/336) with a mean age of 72 years received vasopressor therapy via PIV access, a total of 783 times. ${ }^{[22]}$ The medications used included norepinephrine, dopamine, and phenylephrine. During the study period, $82 \%$ of patients received vasopressor medication via PIV compared to $18 \%$ via CVC. ${ }^{[22]}$ The duration of vasoactive medication infusion via PIV access was $49 \pm 22$ hours. Extravasation occurred in 19 patients (2\%) without any tissue injury following the extravasation protocol. Ninety-five (13\%) patients receiving vasopressor medications through PIV ultimately required a CVC. ${ }^{[22]}$ This evidence-based protocol for peripherally administered vasopressors decreased central line use and the potential complications from the placement of CVCs. An essential part of this nurse-driven protocol's success was the educational process. Before initiation of the protocol, all members of the MICU interdisciplinary team, as well as hospital administration and educators became well-versed on the protocol and expectations of the trial. Patient safety was a priority. Limitations of this study included that the design was single center, observational and a clinical unit trial. It was suggested by the authors that this study be replicated in other types of clinical settings with a larger patient sample.

\section{Discussion}

Key components of this quality improvement project were patient safety, a team approach and skilled monitoring of the PIV site. The critical care nurses were responsible for the line insertion, maintenance and identification of possible extravasation. ${ }^{[22]}$ Administration of norepinephrine, dopamine, or phenylephrine by PIV access was proven safe in this MICU pilot study. Routine placement of CVCs for vasopressor administration decreased. ${ }^{[22]}$ This may equate to fewer CLABSIs, as well as other well recognized complications of central venous access placement.

Critically ill patients often require vasopressors emergently and the requirement of a CVC for administration may delay treatment. This delay may negatively impact patient outcomes related to the patient remaining hemodynamically unstable during catheter insertion. ${ }^{[12]}$ Peripherally inserted catheters that are confirmed with ultrasonography may be a more feasible option in this type of unstable patient. Once vasopressors are infusing via monitored PIV catheters and the patient is stabilized, a CVC can be placed if the clinician deems it necessary. All patients were assessed for the need of a CVC versus PIV access for vasopressor administration by the medical staff and critical care intensivist. Once determined a PIV catheter would be suitable, the protocol was strictly followed and documented to ensure patient safety.

Lastly, the study that utilized this protocol was completed in a single tertiary medical center, which is part of a much larger healthcare system. Since the results of this initial pilot study were positive, this protocol can be expanded to multiple sites in different geographical regions and into other types of intensive care units. This nurse-driven protocol will be initiated at the participating sites once the clinical management team has been educated. Data will be collected prospectively and entered into a standard data collection system for quality and safety assessment.

\section{Implications for clinical practice}

The safe administration of peripheral vasopressors has many implications for nursing practice. CLABSIs lead to increased patient morbidity and mortality. Reducing the incidence of CLABSIs not only improves patient outcomes but can also decrease medical costs. A nurse-driven protocol for peripherally administered vasopressors has significantly decreased central line days and the potential complications from the placement of CVCs. Placing a PIV catheter, which is faster than placing a CVC, allows hemodynamically unstable patients to receive vasopressors sooner than they would otherwise. Therefore, peripheral administration of vasopressors may decrease the amount of time a patient remains hemodynamically unstable.

\section{Conclusion}

The development of this nurse-driven protocol for the safe administration of vasopressors through PIV catheters has demonstrated the importance of using the scientific evidence to develop new standards of care. It is vital that clinical nurses lead the effort to develop evidence-based protocols to improve the quality of patient care, always keeping safety as the top priority. Strict adherence to protocol is essential for the safe administration of vasopressors via PIV access. As more research becomes available, this protocol will need to be revised. It is imperative to look at every individual patient situation and determine the best course of treatment. Practitioners should not consider vasopressor administration to be an automatic indication for CVC insertion.

\section{CONFLicts OF InTEREST Disclosure}

The authors declare no conlicts of interest. 


\section{REFERENCES}

[1] McGee DC, Gould MK. Preventing complications of central venous catheterization. New England Journal of Medicine. 2003; 348(12): 1123-33. https://doi.org/10.1056/NEJMra011883

[2] Zochios V, et al. Peripherally inserted central catheter (PICC)-related thrombosis in critically ill patients. Journal of Vascular Access. 2014; 15(5): 329-37. https : //doi.org/10.5301/jva.5000239

[3] Soni NJ, Reyes LF, Keyt H, et al. Use of ultrasound guidance for central venous catheterization: A national surveyof intensivists and hospitalists. Journal of Critical Care. 2016; 36: 277-283. http: //dx.doi.org/10.1016/j.jcrc2016.07.014

[4] Nuckols TK, et al. Economic Evaluation of Quality Improvement Interventions for Bloodstream Infections Related to Central Catheters: A Systematic Review. JAMA Internal Medicine. 2016; 176(12): 1843-1854. https://doi.org/10.1001/jamainternmed. 2016 .6610

[5] Topal J, et al. Prevention of nosocomial catheter-associated urinary tract infections through computerized feedback to physicians and a nurse-directed protocol. American Journal of Medical Quality. 2005; 20(3): 121-126. https ://doi.org/10.1177/10628606052760 74

[6] Ilott I, Patterson M, Turgoose C, et al. What is protocol-based care? A concept analysis. Journal of Nursing Management. 2006; 14: 544552. PMid:17004965. https://doi.org/10.1111/j.1365-293 4.2006.00703.x

[7] Rycoft-Malone J, Fontenla M, Seers K. Protocol-based care: Impact on roles and service delivery. Journal of Evaluation in Clinical Practice. 2008; 14(5): 867-873. PMid:19018920. https://doi.org/ $10.1111 / j .1365-2753.2008 .01015 . x$

[8] Harbour R, Miller J. A new system for grading recommendations in evidence based guidelines. British Medical Journal. 2001; 323(7308): 334-336. PMid:11498496. https ://doi.org/10.1136/bmj . 32 3.7308 .334

[9] Rycroft-Malone J, Morrell C, Bick D. The research agenda for protocol-based care. Nursing Standard. 2004; 19(6): 33-36. https : //doi.org/10.7748/ns2004.10.19.6.33.c3730

[10] Lewis T, et al. Safety of the Peripheral Administration of Vasopressor Agents. Journal of Intensive Care Medicine. 2017; 885. https://doi.org/10.1177/0885066616686035

[11] Delgado T, et al. Safety of peripheral administration of phenylephrine in a neurologic intensive care unit: A pilot study. Journal of Critical
Care. 2016; 34: 107-110. https://doi.org/10.1016/j.jcrc.2 016.04 .004

[12] Loubani OM, Green RS. A systematic review of extravasation and local tissue injury from administration of vasopressors through peripheral intravenous catheters and central venous catheters. Journal of Critical Care. 2015; 30(3): 653 e9-17. https : //doi .org/10.1 $016 / j \cdot j c r c .2015 .01 .014$

[13] Kahn JM, Kress JP, Hall JB. Skin necrosis after extravasation of low-dose vasopressin administered for septic shock. Critical Care Medicine. 2002; 30(8): 1899-1901. PMid:12163813. https://doi. org/10 . 1097/00003246-200208000-00038

[14] Le A, Patel S. Extravasation of Noncytotoxic Drugs: A Review of the Literature. Annals of Pharmacotherapy. 2014; 48(7): 870-886. https://doi.org/10.1177/1060028014527820

[15] Roth D. Extravasation injuries of peripheral veins a basis for litigation? Journal of Vascular Access Devices. 2003; 8(1): 13-19. https://doi .org/10.2309/108300803775307729

[16] Camp-Sorrell D. Developing extravasation protocols and monitoring outcomes. Journal of Intravenous Nursing. 1998; 21(4): 232-239. PMid:9866539.

[17] Alexander ME. Infusion Nursing Standards of Practice. Journal of Intravenous Nursing. 2000; 23(65): s42.

[18] Chen JL, O'Shea M. Extravasation injury associated with lowdose dopamine. Annals of Pharmacotherapy. 1998; 32(5): 545-548. https://doi.org/10.1345/aph.17184

[19] Joing S, et al. Videos in clinical medicine. Ultrasound-guided peripheral I.V. placement. New England Journal of Medicine. 2012; 366(25): e38. https://doi.org/10.1056/NEJMvcm1005951

[20] Lexicomp-Lexi-Drugs, Phentolamine. 2015. Wolters Kluwer Health, Inc: Hudson, OH. Available from: http://online.lexi.com. Accessed February 20, 2015.

[21] Ricard JD, et al. Central or peripheral catheters for initial venous access of ICU patients: a randomized controlled trial. Critical Care Medicine. 2013; 41(9): 2108-2115. https://doi.org/10.1097/ CCM. Ob013e31828a42c5

[22] Cardenas-Garcia J, et al. Safety of peripheral intravenous administration of vasoactive medication. Journal of Hospital Medicine. 2015; 10(9): 581-585. https : //doi.org/10.1002/jhm. 2394

[23] Reynolds PM, et al. Management of extravasation injuries: a focused evaluation of noncytotoxic medications. Pharmacotherapy. 2014; 34(6): 617-632. https ://doi .org/10.1002/phar.1396 